Research Article

\title{
Design of a Flexible Exhibition Management Information System for Exhibition Services
}

\author{
Bin Hu and Weida Shi 1 \\ Department of Foreign Language \& Tourism, Hebei Petroleum University of Technology, Chengde 067000, China \\ Correspondence should be addressed to Weida Shi; swd1200@m.fafu.edu.cn
}

Received 7 September 2021; Accepted 20 October 2021; Published 19 November 2021

Academic Editor: Rahman Ali

Copyright (c) 2021 Bin Hu and Weida Shi. This is an open access article distributed under the Creative Commons Attribution License, which permits unrestricted use, distribution, and reproduction in any medium, provided the original work is properly cited.

In order to solve the problems of low efficiency of management information systems and low utilization rate of the information resources, this research paper proposes the designs of an exhibition management information system by using a B/S structure (Browser/Server mode). The proposed system is aimed at the analysis of functions and types of exhibitions. The system is comprised of the following three components: the browser-side, server-side, and middle layer. Among them, the browser includes a user management module and exhibition environment visualization module. The server includes an upper computer control module and human-computer interaction module. The middle layer includes the data resource management module, document information management module, exhibition project audit approval and management module, and information scheduling module. Experimental results show that the lowest management efficiency of the system is $96.19 \%$, the highest can reach $98.39 \%$, and the utilization rate of information resources is above $94 \%$, indicating that the system has achieved the design expectation.

\section{Introduction}

Exhibition refers to conference, exhibition, large-scale activities, and other collective commercial or noncommercial activities. Its conceptual connotation refers to mass social activities that are formed by many people gathering together in a certain geographical space to transmit and exchange information regularly or irregularly, systematically or unsystematically. The extension of its concept includes various types of expositions, exhibition and sales activities, conferences of large, medium, and small sizes, cultural activities, and festival activities [1]. Among them, the exhibition with a specific theme refers to the gathering and communication activities of people around a specific theme in a specific time and space. The narrow sense of exhibition only refers to exhibitions and conferences; in the broad sense, exhibition is a general term for conferences, exhibitions, festival activities, and all kinds of industrial/industry-related exhibitions. Conference, exhibition, exposition, and trade fair are the basic forms of exhibition activities; the world exposition is the most typical exhibition activity.
With the continuous improvement of China's comprehensive economic strength, the exhibition industry has been rapidly developed. There are various types of exhibitions, promoting the exchange and development of various fields. Modern exhibition content covers multiple components; the venue is composed of multiple venues with different functions, and the number of participants is large and has different types. At present, the domestic exhibition industry chain has been quite perfect [2].

Exhibition service management is an essential emerging field in the development of the modern service industry, which is at the core of the exhibition industry chain and determines the success or failure of the whole exhibition work. The exhibition industry is the sunrise enterprise in the competitive market. With the improving Internet technology and popularization, the digital and intelligent development has already become the inevitable trend of exhibition industry development; the traditional pattern of convention and exhibition services have been unable to meet the demands of exhibitors' and visitors' service experience; combined with information technology, the innovation 
exhibition service can effectively improve the service efficiency and quality. Therefore, exhibition enterprises should actively explore intelligent innovation paths and optimize and transform exhibition services, so as to promote sustainable development of the exhibition industry [3].

To this end, relevant scholars designed an exhibition industry integration management system based on the "cloud + VR" technology [4]. In the context of the integrated development of "Internet +" and the exhibition industry, emerging technologies such as big data, cloud computing, and artificial intelligence are actively related to the exhibition industry and penetrating effect, in order to find their own new industrial form. As a result, the system integration model as a theoretical basis, based on the analysis of cloud +, VR technology, and exhibition industry development, on the basis of the theory construction, model build, and build fusion architecture, is divided into three aspects: the theoretical framework and research strategy, using the entropy method and coupling coordination degree model, demonstrate its fusion effect and try to induce the coupling coordination problems. It provides a theoretical basis for creating new digital management. In addition, some scholars proposed an intelligent exhibition service management system based on IPA analysis and optimization [5]. The system is based on technical support, user interaction, and performance of the system of three dimensions and 11 indexes of evaluation, and using the IPA method analysis of the present situation of exhibition service platform wisdom, we found users for application compatibility, update information timely, and convenient entry registration and navigation positioning precision indexes such as the value and satisfaction are higher. They are more satisfied with the clear and reasonable interface layout. They are less satisfied with the importance of multilanguage service, responsiveness of communication and interaction, reliability of peripheral recommendation, and patency of Wi-Fi use. They are more satisfied with the importance of privacy and convenience of transaction service. It considers the wisdom of convention and exhibition service management should be further optimized in keeping the application compatibility, update information, and registration; it has the advantages of navigation and positioning at the same time, focusing on strengthening the users' privacy protection, being convenient to provide professional services, giving attention to two or more things such as interface layout, multilingual services, and interactions, and recommending and using $\mathrm{Wi}$ Fi improvement and perfection.

Recently, in [6], a new exhibition management information system is proposed which is simple, easily extendable, and of low cost. It is used for correspondence between the exhibition center and its different stakeholders. Similarly, in [7], an intelligent exhibition guidance service-based system is proposed which makes use of dynamic customer experience. In [8], a central control system in a smart exhibition hall is designed which supports innovation at the application level.

However, in practical application, it is found that the traditional exhibition service management system has some problems such as low management efficiency, low resource utilization rate, and poor system operation stability. In order to effectively alleviate this problem, a new exhibition analysis service management system is designed and put into use.

In this research article, an exhibition management information system is proposed by using a $\mathrm{B} / \mathrm{S}$ structure (Browser/Server mode). This system is comprised of the following three components: the browser-side, server-side, and middle layer. Among them, the browser includes a user management module and exhibition environment visualization module. The server includes an upper computer control module and human-computer interaction module. The middle layer includes the data resource management module, document information management module, exhibition project audit approval and management module, and information scheduling module.

\section{Relevant Analysis of Exhibition Industry}

At present, the origin of exhibition is still being discussed and studied, and there is no unified and positive view. There are roughly "market evolution" theory, "witchcraft etiquette and sacrifice" theory, and "barter" theory. Therefore, we can believe that exhibition develops with the progress of society's economy, politics, and culture and exists and develops perfectly around people's material and spiritual needs [9].

2.1. Functions of Exhibition. Exhibition has powerful economic functions, including connection and transaction function, integrated marketing function, supply and demand regulation function, technology diffusion function, industrial linkage function, and promoting economic integration.

(a) Connection and transaction functions: contact and transaction function exhibition breeds huge business opportunities. The communication function of exhibition is very obvious: the amount of contact is large; the contact area is wide; and the connection effect is good. Therefore, exhibition can provide the organizer, exhibitors, and visitors with the opportunity to contact and communicate with each other [10]. During a limited event period of only a few days, exhibitors often have access to most customers of the entire industry or market, perhaps more than in a year or even years through conventional means such as house visits. In the professional exhibition, the exhibition participants can contact leaders of the competent departments of the industry, experts in the field, existing customers, potential customers, suppliers, agents, users, and other relevant people in various roles, including decision makers and key people, forming high interpersonal contact quality. The elegant atmosphere of the exhibition is conducive to high-quality communication [11].

(b) Integrated marketing function: as an effective marketing platform between enterprises, exhibition provides a bridge and link for enterprises to display products, collect information, negotiate trade, exchange technology, and expand the market. Exhibition is playing an increasingly 
important role in the marketing strategy of enterprises. In developed countries, exhibition marketing has become an important marketing means for many enterprises [12]. At the same time, the exhibition economy is a kind of economic competition. Many suppliers and demanders gather together to directly exchange supply and demand information. This is a market that is close to perfect competition, and the law of market value can play the greatest role [13].

(c) Function of regulating supply and demand: the function of regulating supply and demand exhibition can be regarded as information market, information can be exchanged, and the information of enterprises participating in the products is market information and is an important resource of the market economy.

(d) Technology diffusion function: in the technology diffusion functions, the exhibition organizations are adopting to the innovations in the business processes.

(e) The industrial linkage function: it includes information, components, raw materials, finished goods, and transport links between the exhibition service providers and the organizations.

(f) Economic integration: it reduces the costs of the exhibitors and improves the availability of goods and services to the clients' organizations and individuals.

Information market is the pivot of the economic operation cycle. The exhibition information market reflects the information exchange between supply and demand in a variety of economic relations; it connects the market information provider, market information users, and important production factors such as market information resource applications to promote all kinds of market resources to optimize configuration, effectively stimulate demand, and adjust the supply.

\subsection{Classification of Exhibitions}

(1) According to the nature of exhibition, projects can be divided into trade exhibition projects and consumer exhibition projects:

(a) trade exhibition project is indicating to the manufacturing industry; in business and other industries at the exhibition, exhibitors and visitors are businessmen; exhibitors can be industry manufacturers, traders, wholesalers, distributors, agents, and other related units; visitors are mainly used to invite buyers, while general audiences have been excluded. The ultimate purpose of the exhibition is to reach a deal [14].

(b) Consumer exhibition projects refer to the exhibition activities held for the public. Most of these exhibition projects are of local nature, and the exhibition content is mainly of consumer goods, attracting audiences through mass media such as TELEVISION, radio, newspapers and magazines, and the Internet. The audience is mainly consumers; consumers need to buy tickets to enter. This kind of program attaches great importance to the number of audience. Consumer exhibition is shown in Figure 1.

(c) The main criterion to distinguish exhibition projects from trade or consumption is the composition of the audience, that is, whether the audience is traders or ordinary consumers, rather than the exhibits, that is, industrial products or consumer goods.

(2) According to the content of exhibition projects, they can be divided into comprehensive exhibition projects and professional exhibition projects:

(d) Comprehensive exhibition refers to the exhibition including the whole industry or several industries, also known as horizontal exhibition, such as heavy industry exhibition light industry exhibition

(e) Professional exhibition refers to an exhibition that displays a certain industry or even a certain product such as watch exhibition [15]

In recent years, the rapid development of the exhibition industry has shown that it should not be an underestimated industry; in the more and more mature market economy environment, how to supervise and guide the standard development of the exhibition industry has become a key problem to be solved. The original multihead examination and approval system no longer meets the development needs of the exhibition industry, and only indirect regulation and guidance through regulations and industry standards can guarantee its benign development [16].

\section{Exhibition Analysis Service Management System Design}

On the basis of the abovementioned analysis, this study designs an exhibition analysis service management system for comprehensive exhibition projects, which mainly uses the $\mathrm{B} / \mathrm{S}$ structure. This paper constructs the system data structure, function modules, and operation flow. The B/S structure not only simplifies the workload of the system but also supports the distributed processing of exhibition data and information, which effectively reduces the cost of information management. At the same time, clients with a three-tier $\mathrm{B} / \mathrm{S}$ architecture are more versatile, less dependent on the application environment, and can effectively provide system management efficiency. The $\mathrm{B} / \mathrm{S}$ structure is shown in Figure 2.

In the abovementioned $\mathrm{B} / \mathrm{S}$ structure, the exhibition analysis service management system can be divided into three parts: the browser-side, server-side, and middle layer. The detailed analysis is carried out as follows:

(1) Browser layer: this layer is combined with the data structure of exhibition resources, and it can provide 


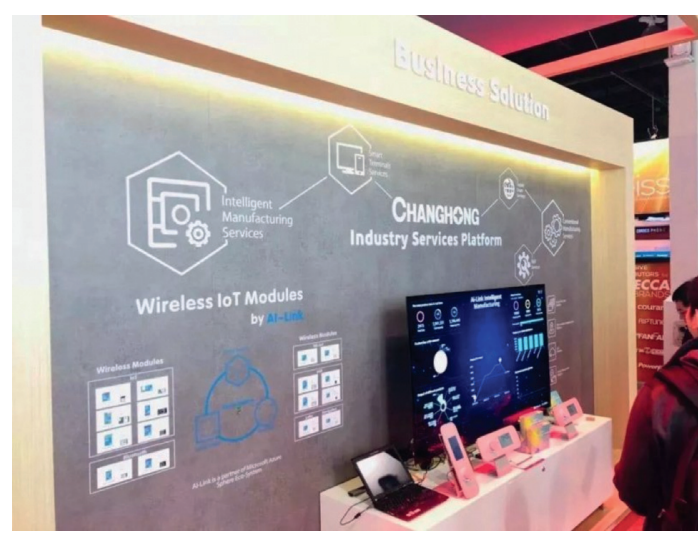

FIgURE 1: Scene of consumer exhibition.

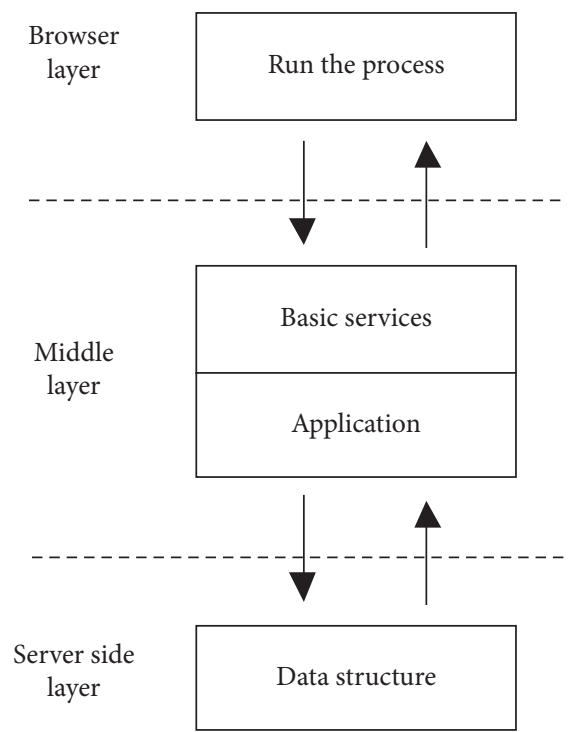

Figure 2: B/S structure (Browser/Server mode) diagram.

data services, transmit service results to the middle layer, and store data on the server side including the user management module and exhibition environmental visualization module.

(2) Server-side layer: this layer mainly runs various application services, can receive the browser side of the information management request, and can display the request processing results. In this study, the corresponding running process is embedded in the server and the connection between the system modules is established including the upper computer control module and human-computer interaction module.

(3) Middle layer: this layer mainly realizes data management functions and can receive user requests, send requests to the server, and then, return service results to users. It includes the data resource management module, document information management module, exhibition project audit and management module, and information scheduling module.

\subsection{Browser}

3.1.1. User Management Module. The user management module provides the user information management of the exhibition analysis service management system, including user registration, user login, user authority management, user information modification, and user level modification.

\subsubsection{Exhibition Environment Visualization Module. This} study analyzes the types and behavioral patterns of participants and possible abnormal events by combining time, space, speed, and other attributes, so as to help the exhibition organizers review the overall situation of the exhibition more easily and effectively, have insight into the spatial behavior of participants, find relevant deficiencies, and improve the management level in the future. Therefore, the design of views in this paper mainly follows the following two principles: views accurately display the complex information contained in data; views are clear and intuitive, reducing the users' burden of understanding and cognition of data.

The exhibition environment visualization module is mainly composed of a three-dimensional map, global situation map, track playback map, track Gantt chart, speed Gantt chart, duration Gantt chart, control panel, and line chart of population change [17]. Due to the complexity of data, it is difficult to directly display the results by machine processing data to meet the needs of users. Therefore, a multiview association analysis is necessary, and rich interaction can also make users enjoy the fun of exploration. This system provides two interactive entrances, namely, a global situation map and control panel. The visualization process is as follows:

(1) Click the "Play" button to help users dynamically perceive the global situation.

(2) Pause the animation, select the target point of interest on the map, and then, perform Steps 3-7 or 8 .

(3) The Gantt chart of "Stay Time" shows the stay time of the personnel who enter the target point (one or more points, or an area) on that day.

(4) Select the IDsyou are interested in on the Gantt Chart of Stay Duration. The control panel will display these selected IDs.

(5) Perform the query when + WHO $\longrightarrow$ WHERE+ speed to display the speed and track of the selected ID in the "Speed Gantt chart" and "Track Gantt Chart," and then, perform Step 6 or Step 7.

(6) Feedback the coordinate points and time of interest to the map.

(7) Select the ID that is interested in the "Track Gantt chart" and playback its track dynamically in the "Track Playback Map."

(8) The control panel displays the ID of the target point at the moment, and then, perform Steps 5-7.

(9) Execute when + where $\longrightarrow$ who + when query. 
(10) Show the relationship between the number of people and the time of the selected venue in the "Number of people Change Line Chart."

(11) Repeat Steps 3-7.

(12) Enter the ID of the person you are interested in in the control panel.

(13) Repeat Steps 5-7.

3.2. Server Side. The exhibition service management system of the server side includes the PC control module and human-computer interaction module, on this basis, the output of the set system bus protocol, combining crosscompilation control technology for a PC control, and an intelligent design of human-computer interaction, so as to build the server running model, and uses the upper machine communication method for network design.

At the same time, considering the working characteristics of the exhibition analysis service management system, the protocol communication and interactive process output transformation control are designed by the database scheduling method, and the bus is developed and designed by the ITU-656 PPI pattern recognition method.

3.3. The Middle Tier. This section describes different modules and components of the middle tier.

3.3.1. Data Resource Management Module. This module adopts a parallel input/output control method to manage exhibition data resources. Firstly, the statistical feature analysis method is adopted to carry out the adaptive scheduling and feature extraction of information of the exhibition analysis service system, and the distributed scheduling detection statistics are obtained as follows:

$$
z=(a+b) \times \frac{\chi \delta}{2 c^{2}} .
$$

Among them, $a$ represents the forward scheduling statistical features, $b$ represents the reverse scheduling statistical features, $c$ represents a random exhibition analysis service data to be scheduled, $\delta$ represents a random exhibition analysis service data to be scheduled, and $\chi$ represents the standard scheduling coefficient.

On the basis of obtaining detection statistics, the statistical distribution model of data resources is constructed, and the correlation characteristics between data are obtained by using the time-delay equalization control method:

$$
A=\int_{U_{\min }}^{U_{\max }} \frac{f+b}{\varepsilon z} .
$$

Among them, $U_{\max }$ represents the upper limit of data carrying capacity of the exhibition analysis service system, $U_{\min }$ represents the lower limit of data carrying capacity of the exhibition analysis service system, and $\varepsilon$ represents the standard definition [18].

Scattered in the feature subspace of test scheduling and fuzzy data, on this basis, using the multiple queue scheduling method, the exhibition data analysis service system fuzzy decision model is established, and a unix-like operating data clustering and cross-compiling control scheduling process fusion and the exhibition service system output statistical characteristic of data resource management are as follows:

$$
E=\sum_{i=1}^{N} x_{i}(f+b) \times A
$$

\subsubsection{Document Information Management Module. The} document information management module is mainly responsible for the information management of the document class. In the document information management module, the two core functions are document delivery and document approval, and efficient document delivery and document approval are also the basis to ensure the normal operation of the system [19].

First of all, relevant staff needs to input new exhibition resource information into the system, so that managers can query the new information in real time. At the same time, information modeling of exhibition resources is also needed to ensure the efficiency and practicability of information management through the establishment of a database.

In the exhibition resource database, the data are divided into spatial data and attribute data, and then, the data matrix is constructed and expressed as follows:

$$
S=\left[\begin{array}{cccc}
s_{11} & s_{12} & \cdots & s_{1 m} \\
s_{21} & s_{21} & \cdots & s_{2 m} \\
\vdots & \vdots & \ddots & \vdots \\
s_{n 1} & s_{n 2} & \cdots & s_{n m}
\end{array}\right],
$$

where $m$ represents the total amount of exhibition data resources and $n$ represents the attribute elements of exhibition data resources. In order to improve system management efficiency and reduce system management cost, the abovementioned data matrices are normalized and the final data resource information storage management matrix is obtained as follows:

$$
J=D \times \partial \times S,
$$

where $\partial$ represents the normalized coefficient and $D$ represents the Euclidean distance of two points in a two-dimensional image.

\subsubsection{Exhibition Project Audit Approval and Management} Module. The exhibition project audit initiation and management module of the exhibition analysis service management system contains two executive roles: an ordinary user and administrator user. In addition, the two user roles have different operation and processing rights for many application functions, such as project initiation, project deletion, project modification, and audit scheme setting. Among them, the application for exhibition project approval refers to the process of information input, project plan upload, project application form filling, etc., carried out by 
the project initiator for the exhibition project. During the implementation of the application instruction for project approval, all project objects must maintain an independent connection state. When implementing the modification instruction of exhibition projects, an administrator user can face multiple audit objects of different exhibition projects at the same time and establish multiple new project files while summarizing the projects to be audited. Ordinary users have the right to upload project application forms and project plan documents related to the audit business of exhibition projects on the application information interface, and the "batch project approval" clause should be established for individual project objects, and mining association rules can be established based on this.

The operation principle of exhibition project audit is shown in Figure 3.

On this basis, the management function of exhibition project audit files mainly has four basic execution requirements of query, increase, delete, and modify, which can match different operation rights of exhibition project audit and management for administrator users and ordinary users, respectively. Administrators or ordinary users first log in to the system operation interface with the help of the BP neural network and then click the file management button to enter the system's core file management interface. Logged-in users can select relevant documents of the exhibition project they want to view according to their personal query needs and then transmit the final query results back to the main control interface of the system by means of a feedback channel. In the following execution time, users who have logged in can download the exhibition project documents to be audited to the local storage environment according to their personal requirements, so as to generate long-term archive files to facilitate the retrieval and application of system association rules.

3.3.4. Information Scheduling Module. In this module, the decision tree model is used to schedule the relevant exhibition analysis service information, the fuzzy feature quantity is extracted from the information, and then, the edge pixel feature reconstruction method is used to complete the adaptive scheduling. Firstly, the statistical feature distribution set of exhibition analysis service information obtained is defined as $T$; then,

$$
T=x_{i} \times\left(\frac{\mu \beta}{q}\right) \text {. }
$$

Among them, $x_{i}$ represents the training data, $\alpha$ represents the spatial clustering function of the information scheduling process, $\beta$ represents the ambiguity of exhibition analysis service information, and $q$ represents the correlation model. On this basis, the piecewise linear management method is adopted for information scheduling, and the quantized feature distribution set is as follows:

$$
T^{\prime}=\left\{x_{1} T, x_{2} T, \ldots, x_{i} T, \ldots x_{n} T\right\}^{d},
$$

where $d$ represents the number of iterations. A $1 \times \mathrm{N}$-dimensional matrix was used to determine the time window value $\mathrm{N}$ of exhibition analysis service information scheduling, and the multidimensional information entropy reconstruction method was used to construct the Probit multiple regression analysis model and carry out information fusion. Firstly, the edge quantization feature distribution set is

$$
B=\min _{0 \leq d \leq N}\left\{T^{\prime}\right\} .
$$

On this basis, the linear random equalization regulation method is adopted to fuse the exhibition analysis service information, and the association rule feature quantity of information data is extracted. Thus, the transmission learning function of exhibition analysis service information scheduling is as follows:

$$
F=\frac{\sum_{i=1}^{N}\left(B / T^{\prime}\right)}{N(N-1) / 2} .
$$

\section{Experimental Verification and Analysis}

In order to verify the practical application effect of the exhibition analysis service management system designed above, the following experiments are designed. The experimental environment is as follows: 2.10ghz AMD R5CPU (256G memory), Windows10 operating system, and the management program is implemented on the VS2015 platform using $C++$ programming language.

In order to avoid too many single experimental results, the traditional exhibition industry integration management system based on "cloud + VR" technology and the intelligent exhibition service management system based on IPA analysis and optimization are taken as the comparison systems to complete performance verification together with the system in this paper. The selected indexes are as follows:

(1) System management efficiency: system management efficiency can directly reflect the actual application effect of different systems. The higher the management efficiency is, the stronger the statistical management ability of the system is for the exhibition analysis service information and the more helpful it is to realize the intelligent utilization of exhibition management.

(2) Utilization rate of information resources: information resource utilization can indirectly reflect the information management effect of different systems. The higher the information resource utilization is, the better the system management is.

First, the management efficiency of different system pairs is tested, and the results are shown in Table 1.

By analyzing the experimental data in Table 1 , it can be seen that the management efficiency of each system will change as the number of experiments increases due to the different management modes adopted by the three systems. The management efficiency of the three traditional systems is below 95\%. The lowest management efficiency of the system in this paper is $96.19 \%$, and the highest can 


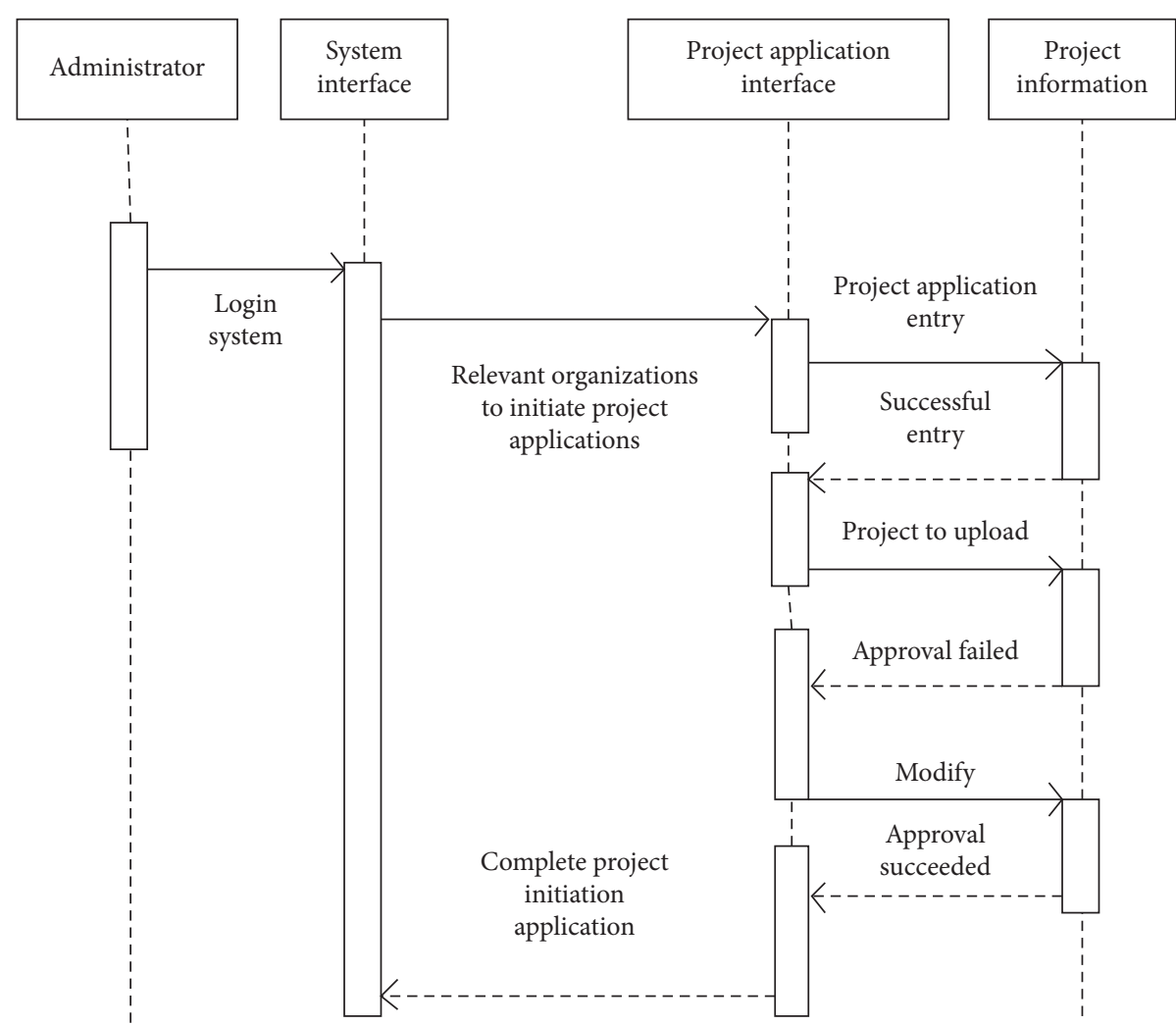

FIGURE 3: Operation principle of the audit project initiation module of the exhibition project.

TABLE 1: Comparison results of the management efficiency of different systems.

\begin{tabular}{lccc}
\hline $\begin{array}{l}\text { Number of } \\
\text { experiments/times }\end{array}$ & $\begin{array}{c}\text { The proposed } \\
\text { system }\end{array}$ & $\begin{array}{c}\text { System management efficiency/\% } \\
\text { Exhibition industry integration management } \\
\text { system based on "cloud + VR" technology }\end{array}$ & $\begin{array}{c}\text { Intelligent exhibition service management } \\
\text { system based on IPA analysis and optimization }\end{array}$ \\
\hline 10 & 97.52 & 94.74 & 93.52 \\
20 & 96.19 & 92.47 & 91.52 \\
30 & 98.39 & 93.25 & 89.22 \\
40 & 98.02 & 91.25 & 88.20 \\
50 & 97.17 & 91.66 & 92.87 \\
\hline
\end{tabular}

reach $98.39 \%$, indicating that the stronger the statistical management ability of the system in this paper for exhibition analysis service information, the more helpful it is to realize the intelligent utilization of exhibition management.

In order to further verify the application results of different systems, the utilization rate of information resources is taken as the verification index to carry out performance tests for different systems, and the results are shown in Figure 4.

Analysis of the experimental data in Figure 4 shows that the utilization rate of information resources of different systems will change with the change of the number of test objects. After the application of the system in this paper, the utilization rate of information resources is above $94 \%$, up to $97 \%$, obviously higher than that of the other two traditional systems, indicating that the management effect of the system in this paper is better.

\section{Related Suggestions}

In order to effectively improve the quality of analysis, service, and management of the exhibition industry, this study puts forward the following suggestions.

5.1. To Improve the Exhibition Database. Exhibition service is not simply to build a database, but through the summary and classification of database information for different applications. Exhibition service should strengthen the construction and perfection of a database and build a database with consumers, exhibitors, organizers, competitors, and other data subjects, which is also an important content of modern exhibition. At the same time, exhibition service enterprises should strengthen the analysis and sorting of a database and provide targeted data analysis for customers, so as to improve the quality of exhibition service. 


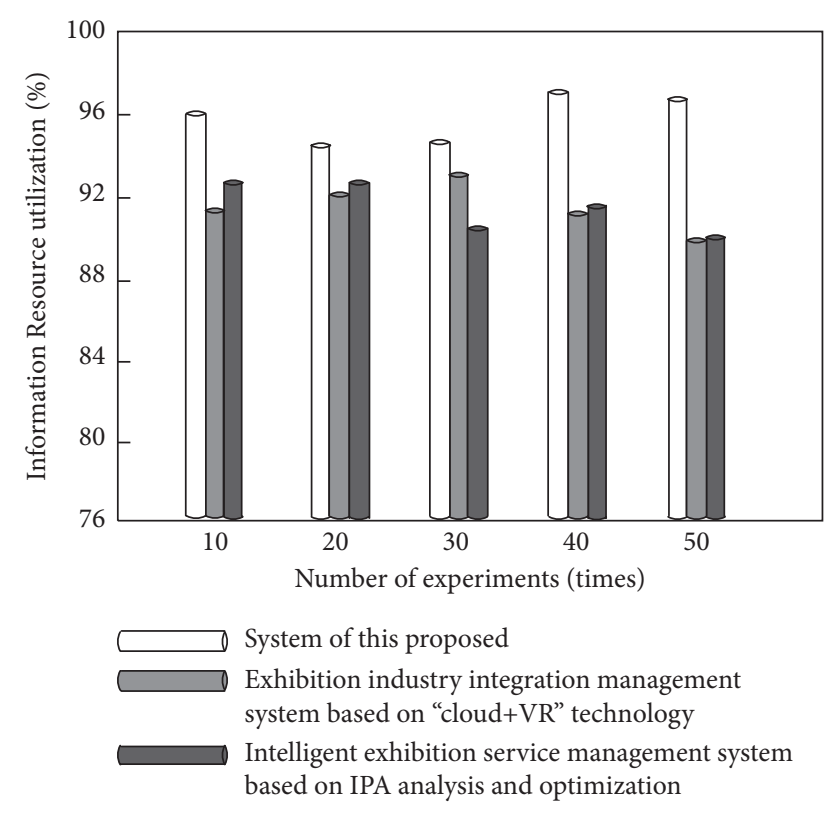

FIgURE 4: Comparison results of information resource utilization of different systems.

5.2. Strengthening the Analysis and Use of Data. Data analysis and application are the important links of current exhibition services. First, the accumulated data should be summarized and sorted out, the potential value of the data should be mined, the value should be fed back to the service, and the optimization effect should be tracked, and then, further optimization and upgrading should be carried out to realize the sustainable development of the exhibition service. The second is to strengthen the inspection and management of cloud platform data, through face recognition, VR, and other technologies to record and analyze the data information of exhibitors, to provide a basis for the development of accurate marketing strategy, and to provide a strong guarantee for the order and safety work of the exhibition site. Third, the ultimate purpose of big data application is to improve the level of exhibition service, so we should make full use of data information to improve the quality of exhibition service and provide customers with more optimized user experience.

5.3. Innovation of the Exhibition Service System. First of all, the exhibition service system should establish a customeroriented service concept, pay attention to user experience, and optimize and upgrade through mining customer demand data. Secondly, modern exhibition services need the most advanced technology as the driver, such as Internet of things technology and AI artificial intelligence technology, through intelligent terminals to provide customers with more intelligent exhibition experience. Thirdly, the content of exhibition services should be constantly innovated, such as human-computer interaction and virtual exhibition hall, and customized services should be carried out according to customer needs.

5.4. Strengthening the Construction of Talent Teams. Talent is the cornerstone of exhibition service innovation. Therefore, it is necessary to actively introduce and cultivate intelligent and high-quality talents, strengthen universityenterprise cooperation, promote the development and construction of intelligent exhibition specialty, strengthen the training and ability improvement of existing talents, and improve the overall quality of talent team, so as to provide powerful guarantee for the innovation of exhibition service.

5.5. Focusing on Customer Needs and Products. For enterprises and individuals participating in exhibition activities, the purpose of participating in the exhibition is different. Some are to go through the process, and some are to collect industry information, secure customers, etc. The exhibition service system should have a thorough understanding of the real needs of customers, develop personalized programs for customers, and provide targeted services. In addition, products are an important content of exhibition projects, and the performance of products is an important factor of the quality of exhibition services. Therefore, exhibition services should pay attention to the cutting-edge trends of exhibition products, dig into the connotation of products, and make adequate preparations.

5.6. Building an Intelligent Exhibition Service Platform. First of all, the exhibition service system should actively build and improve the information service platform, so that the audience can understand the exhibition information and obtain relevant services through their intelligent terminal devices anytime and anywhere. The exhibition service system should constantly improve and innovate the functions of the exhibition service platform, provide diversified platform communication methods, and develop online exhibition functions to improve the effect of exhibition publicity. Secondly, the exhibition service system should strengthen the cooperation with government departments, realize the integration of government information and service platform through the Internet of things technology, further improve the exhibition publicity, strengthen the organic combination of venues and service platform, promote the construction of smart venues, and improve the intelligent exhibition service.

\subsection{Strengthening Innovation in Exhibition Marketing.}

The essence of exhibition service is sales, and the product is service. With the continuous development of the times, people pay more attention to service experience, satisfaction, and appreciation of sex; the conference and exhibition service systems should make full use of modern technology and their own advantages, optimize the service functions, to provide customers with customized services, through long service cycle at the same time, strengthen communication with customers and familiar degree increases way to realize the appreciation of convention and exhibition services, relying on big data and e-commerce to expand marketing channels, and then, improve the benefits of exhibition projects.

5.8. To Enhance the Diversity of Communication Modalities. The exhibition service system functions through the media, the official web, web pages, and live in a variety of ways to 
carry out propaganda and exhibition promotion trend of universal participation, and at the same time, it should make full use of the Internet of things technology, artificial intelligence technology, and so on to strengthen the interactive experience, to give people the feeling of the scene, and help make exhibition information promulgator of the participants.

5.9. Honest Service and Building Brand Effect. First of all, the exhibition service management should establish the awareness of sincere service and brand service, improve the professional ethics of employees, and provide customers with better exhibition service. Secondly, the exhibition service system should make full use of big data technology to conduct customer data mining and analysis and provide the most appropriate and preferential service packages according to consumers' preferences, so as to gradually build a good brand reputation and promote the sustainable development of enterprises through building brand effect.

5.10. Strengthening the Construction of Supporting Facilities to Realize the Interconnection of Everything. The exhibition service system at the same time strengthens the construction of professional services and infrastructure and strengthens the contact and cooperation and the surrounding industry through the cloud data platform for convention and exhibition services, catering, accommodation, and other supporting facilities' resource integration, realizes the exhibition "one-stop service," to further improve the exhibition service efficiency and quality, and boosts regional economic development.

\section{Conclusions}

Based on the analysis of exhibition functions and types, this paper has discussed the design of an exhibition analysis service management system which uses the B/S structure. To validate the proposed system, experimental results are generated which show that the problem of exhibition centers is effectively solved. It has resolved the problems of low system management efficiency and low utilization rate of information resources existing in traditional systems. Experimental results show that the lowest management efficiency of the system is $96.19 \%$, the highest can reach $98.39 \%$, and the utilization rate of information resources is above $94 \%$, indicating that the system has achieved the design expectation.

\section{Data Availability}

The data used to support the findings of this study are available from the corresponding author upon request.

\section{Conflicts of Interest}

The authors declare no conflicts of interest.

\section{References}

[1] K. V. Simonov, "Economic exchanges management in the exhibition bilateral market," Management Science, vol. 10, no. 1, pp. 22-35, 2020.

[2] C. H. Lee, Q. Y. Li, Y. C. Lee, and C. W. Shih, "Service design for intelligent exhibition guidance service based on dynamic customer experience," Industrial Management \& Data Systems, vol. 121, no. 6, pp. 66-78, 2020.

[3] I. G. Filippova, K. I. Derevyanko, E. G. Karpova, and L. V. Khoreva, "Current state of the convention and exhibition market and event services: problems and development trends in a metropolis," Economics and Management, vol. 26, no. 10, pp. 1136-1147, 2020.

[4] B. Xv, "Research on the integration model and Mechanism of "“Cloud +VR"“ technology and exhibition industry," Journal of Shanxi Datong University(Natural Science Edition), vol. 37, no. 4, pp. 38-42, 2021.

[5] X. Liu, L. J. Xv, and X. H. Yang, "IPA analysis and optimization strategy for the operation of intelligent exhibition service platform," Journal of Xiamen University of Technology, vol. 29, no. 2, pp. 52-58, 2021.

[6] Y. Haiying, "Exhibition management information system design and implementation,"vol. 4, pp. 633-636, in Proceedings of the 2010 The 2nd International Conference on Computer and Automation Engineering (ICCAE), vol. 4, IEEE, Singapore, 2010 Feb 26.

[7] C. H. Lee, Q. Li, Y. C. Lee, and C. W. Shih, "Service design for intelligent exhibition guidance service based on dynamic customer experience," Industrial Management \& Data Systems, vol. 121, 2020 Nov 24.

[8] J. Ding and J. Zhang, "Design and application innovation of central control system in smart exhibition Hall," in Proceedings of the 2020 3rd International Conference on $\mathrm{Hu}$ manities Education and Social Sciences (ICHESS 2020), pp. 1117-1120, Atlantis Press, Chengdu, China, 2020 Dec 16.

[9] B.-S. Kim, "A study on improvement of the preliminary feasibility study for the construction of exhibition and convention centers," Korea Trade Exhibition Review, vol. 14, no. 1, pp. 69-100, 2019.

[10] Y. Y. Yang, C. Lei, X. X. Lu, and J. Zhu, "The opening process and development strategy of China's exhibition industry," Macroeconomic Management, vol. 6, no. 3, pp. 78-83, 2019.

[11] A. Ulyanov, E. Genadieva, and M. Draganov, "Management of exhibition and convention events," in Proceedings of the DTMIS "20: International Scientific Conference - Digital Transformation on Manufacturing, November 2020.

[12] A. Shaikh, S. Tiwari, and N. Shaikh, "Information technology service management system," Software Engineering Process Technology, vol. 16, no. 3, pp. 97-105, 2021.

[13] M. Su, Y. Deng, R. Guo, and Q. Wang, "Research on the management model of $3 \mathrm{D}$ application visualization platform," Journal of Chinese Computer Systems, vol. 40, no. 4, pp. 808-813, 2019.

[14] B. Ghita Aditya, "Exhibition service quality and its influence to exhibitor satisfaction," in Proceedings of the 1st International Conference One Belt, One Road, One Tourism (ICOBOROT 2018), 2019.

[15] M. F. Esen and E. Kocabas, Personal Data Privacy and Protection in Meeting, Incentive, Convention and Exhibition (MICE) Industry, USA, 2019.

[16] L. U. Grace, "Research on operation and management of exhibition venues based on exhibitors" satisfaction," 
Pioneering with ence \& Technology Monthly, vol. 32, no. 3, pp. 45-48, 2019.

[17] L. Liu, H. Hu, and T. Yang, "Visual analysis system for exploring spatio-temporal exhibition data," Journal of Computer Applications, vol. 40, no. 9, pp. 2719-2727, 2020.

[18] S. Oh, J. Han, J. Han, D. Jung, and J. Lee, "A study on the effect of the function of the exhibition and convention center on the effectiveness," Journal of Tourism Management Research, vol. 24, no. 6, pp. 347-369, 2020.

[19] H. Ge, X. Zou, and Y. Yi, "Exhibition interactive design research based on leap motion technology," Computer Simulation, vol. 35, no. 2, pp. 459-462, 2018. 